\title{
Nontrivial solutions for an integral boundary value problem involving Riemann-Liouville fractional derivatives
}

\author{
Zhengqing $\mathrm{Fu}^{1}$, Shikun Bai ${ }^{2}$ Donal $\mathrm{O}^{\prime}$ Regan ${ }^{3}$ and Jiafa $\mathrm{Xu}^{2 *}$
}

\section{"Correspondence:}

xujiafa292@sina.com

${ }^{2}$ School of Mathematical Sciences,

Chongqing Normal University,

Chongqing, China

Full list of author information is

available at the end of the article

\begin{abstract}
In this paper using topological degree we study the existence of nontrivial solutions for a fractional differential equation involving integral boundary conditions. Here, the nonlinear term may be sign-changing and may also depend on the derivatives of the unknown function.
\end{abstract}

Keywords: Fractional integral boundary value problems; Nontrivial solutions; Topological degree

\section{Introduction}

We study the existence of nontrivial solutions for the following integral boundary value problem involving Riemann-Liouville fractional derivatives:

$$
\left\{\begin{array}{l}
D_{0+}^{\beta} D_{0+}^{\alpha} u(t)=f\left(t, u(t), u^{\prime}(t),-D_{0+}^{\alpha} u(t)\right), \quad 0<t<1, \\
u(0)=u^{\prime}(0)=0, \quad u^{\prime}(1)=\int_{0}^{1} g(t) u^{\prime}(t) d t, \\
D_{0+}^{\alpha} u(0)=D_{0+}^{\alpha+1} u(0)=0, \quad D_{0+}^{\alpha+1} u(1)=\int_{0}^{1} h(t) D_{0+}^{\alpha+1} u(t) d t,
\end{array}\right.
$$

where $\alpha, \beta \in(2,3]$ are two real numbers, $D_{0+}^{\alpha}, D_{0+}^{\beta}$ are the Riemann-Liouville fractional derivatives, and $f \in C\left([0,1] \times \mathbb{R}^{3}, \mathbb{R}\right)(\mathbb{R}=(-\infty,+\infty))$. Moreover, the functions $g, h$ are defined on $[0,1]$ and satisfy the condition:

(H0) $g, h \geq 0$ with $\int_{0}^{1} g(t) t^{\alpha-2} d t \in[0,1)$, and $\int_{0}^{1} h(t) t^{\beta-2} d t \in[0,1)$.

Fractional-order problems arise naturally in engineering and scientific disciplines such as physics, biophysics, chemistry, control theory, signal and image processing, and aerodynamics; we refer the reader to $[1-3]$. For example, in $[4,5]$ the authors introduced a fractional-order model of infection of $\mathrm{CD} 4^{+} \mathrm{T}$-cells, and the system takes the following form:

$$
\left\{\begin{array}{l}
D^{\alpha_{1}}(T)=s-K V T-d T+b I \\
D^{\alpha_{2}}(I)=K V T-(b+\delta) I \\
D^{\alpha_{3}}(I)=N \delta I-c V
\end{array}\right.
$$

(c) The Author(s) 2019. This article is distributed under the terms of the Creative Commons Attribution 4.0 International License (http://creativecommons.org/licenses/by/4.0/), which permits unrestricted use, distribution, and reproduction in any medium, provided you give appropriate credit to the original author(s) and the source, provide a link to the Creative Commons license, and indicate if changes were made. 
where $D^{\alpha_{i}}$ are fractional derivatives, $i=1,2,3$. Many results on the existence and multiplicity of solutions (or positive solutions) of nonlinear fractional differential equations can be found for example in [6-37] and the references therein. In [6-11, 19, 20, 26], the authors used the fixed point index theory to study the existence of (positive) solutions for various boundary value problems of fractional differential equations, for example, Bai in [6] obtained positive solutions for the nonlocal fractional-order differential equation boundary value problem

$$
\left\{\begin{array}{l}
D_{0+}^{\alpha} u(t)+f(t, u(t))=0, \quad 0<t<1 \\
u(0)=0, \quad \beta u(\eta)=u(1)
\end{array}\right.
$$

where $1<\alpha \leq 2,0<\beta \eta^{\alpha-1}<1,0<\eta<1, f$ is continuous on $[0,1] \times \mathbb{R}^{+}$and satisfies the conditions

$$
(\mathrm{H})_{\mathrm{B} 1} \quad \liminf _{u \rightarrow 0^{+}} \frac{f(t, u)}{u}>\lambda_{1}, \quad \limsup _{u \rightarrow+\infty} \frac{f(t, u)}{u}<\lambda_{1}, \quad \text { uniformly on } t \in[0,1],
$$

and

$$
(\mathrm{H})_{\mathrm{B} 2} \quad \liminf _{u \rightarrow+\infty} \frac{f(t, u)}{u}>\lambda_{1}, \quad \limsup _{u \rightarrow 0^{+}} \frac{f(t, u)}{u}<\lambda_{1}, \quad \text { uniformly on } t \in[0,1]
$$

where $\lambda_{1}$ is the first eigenvalue corresponding of the relevant linear operator. These conditions can also be found in some integer-order differential equations; we refer the reader to [38-52] and the references therein. Integral boundary conditions arise in thermal conduction problems, semiconductor problems and hydrodynamic problems (see [11]) and we refer the reader to $[10,11,18,19,26,28-30,33,34,39,43,50-52]$ and the references therein. In [26] the authors studied the integral boundary value problem of the nonlinear Hadamard fractional differential equation

$$
\left\{\begin{array}{l}
D_{\beta}\left(\varphi_{p}\left(D^{\alpha} u(t)\right)\right)=f(t, u(t)), \quad 1<t<e, \\
u(1)=u^{\prime}(1)=u^{\prime}(e)=0, \quad D^{\alpha} u(1)=0, \quad \varphi_{p}\left(D^{\alpha} u(e)\right)=\mu \int_{1}^{e} \varphi_{p}\left(D^{\alpha} u(t)\right) \frac{d t}{t},
\end{array}\right.
$$

where $\alpha \in(2,3], \beta \in(1,2]$ and $D^{\alpha}, D^{\beta}$ are Hadamard fractional derivatives.

Many papers in the literature also considered sign-changing nonlinearity problems; see $[4,5,7-9,13-19,22-30,34,35,38-52]$ and the references therein. In [15], the authors studied the fractional differential equation with a singular decreasing nonlinearity and a $p$-Laplacian operator:

$$
\left\{\begin{array}{l}
-D_{0+}^{\alpha}\left(\varphi_{p}\left(-D_{0+}^{\gamma} z\right)\right)(x)=f(x, z(x)), \quad 0<x<1, \\
z(0)=0, \quad D_{0+}^{\gamma} z(0)=D_{0+}^{\gamma} z(1)=0, \quad z(1)=\int_{0}^{1} z(x) d \chi(x) .
\end{array}\right.
$$

Using a double iterative technique, they showed that the above problem has a unique positive solution, and from an iterative technique, they established an appropriate sequence, which converges uniformly to the unique positive solution.

In this paper we use topological degree theory to consider the existence of nontrivial solutions for (1.1). The novelty is twofold: (1) the nonlinearity depends on the unknown 
function $u$ and its integer-order, fractional-order derivatives $u^{\prime},-D_{0_{+}}^{\alpha} u,(2)$ the nonlinearity can be unbounded on $[0,1] \times \mathbb{R}^{3}$, which improves results on semipositone problems (i.e., boundedness from below); see [5, 21, 42, 43, 45].

\section{Preliminaries}

We present some definitions and notations from fractional calculus theory involving Riemann-Liouville fractional derivatives; for details see the books [1-3].

Definition 2.1 (see [1-3]) The Riemann-Liouville fractional derivative of order $\alpha>0$ of a continuous function $f:(0,+\infty) \rightarrow(-\infty,+\infty)$ is given by

$$
D_{0+}^{\alpha} f(t)=\frac{1}{\Gamma(n-\alpha)}\left(\frac{\mathrm{d}}{\mathrm{d} t}\right)^{n} \int_{0}^{t}(t-s)^{n-\alpha-1} f(s) d s
$$

where $n=[\alpha]+1,[\alpha]$ denotes the integer part of number $\alpha$, provided that the right side is pointwise defined on $(0,+\infty)$.

Definition 2.2 (see [1-3]) The Riemann-Liouville fractional integral of order $\alpha>0$ of a function $f:(0,+\infty) \rightarrow(-\infty,+\infty)$ is given by

$$
I_{0+}^{\alpha} f(t)=\frac{1}{\Gamma(\alpha)} \int_{0}^{t}(t-s)^{\alpha-1} f(s) d s
$$

provided that the right side is pointwise defined on $(0,+\infty)$.

Lemma 2.3 (see [5, Lemma 2.3]) Let $\alpha>0$, then, for $u, D_{0+}^{\alpha} u \in C(0,1) \cap L(0,1)$, we have

$$
I_{0+}^{\alpha} D_{0+}^{\alpha} u(t)=u(t)+c_{1} t^{\alpha-1}+c_{2} t^{\alpha-2}+\cdots+c_{N} t^{\alpha-N}, \quad \text { for some } c_{i} \in \mathbb{R}, i=1,2, \ldots, N,
$$

where $N$ is the smallest integer greater than or equal to $\alpha$.

Lemma 2.4 Suppose that $(\mathrm{H} 0)$ holds. If let $-D_{0+}^{\alpha} u:=v$, then the fractional boundary value problem

$$
\left\{\begin{array}{l}
-D_{0+}^{\alpha} u(t)=v(t), \quad 0<t<1, \\
u(0)=u^{\prime}(0)=0, \quad u^{\prime}(1)=\int_{0}^{1} g(t) u^{\prime}(t) d t
\end{array}\right.
$$

can be transformed into its equivalent Hammerstein integral equation, which takes the form

$$
u(t)=\int_{0}^{1} G_{1}(t, s) v(s) d s
$$

where

$$
G_{1}(t, s)=\widetilde{G}_{1}(t, s)+\frac{t^{\alpha-1}}{1-\int_{0}^{1} g(t) t^{\alpha-2} d t} \int_{0}^{1} g(t) \widetilde{G}_{2}(t, s) d t,
$$




$$
\begin{aligned}
& \widetilde{G}_{1}(t, s)=\frac{1}{\Gamma(\alpha)} \begin{cases}t^{\alpha-1}(1-s)^{\alpha-2}-(t-s)^{\alpha-1}, & 0 \leq s \leq t \leq 1, \\
t^{\alpha-1}(1-s)^{\alpha-2}, & 0 \leq t \leq s \leq 1,\end{cases} \\
& \widetilde{G}_{2}(t, s)=\frac{1}{\Gamma(\alpha)} \begin{cases}t^{\alpha-2}(1-s)^{\alpha-2}-(t-s)^{\alpha-2}, & 0 \leq s \leq t \leq 1, \\
t^{\alpha-2}(1-s)^{\alpha-2}, & 0 \leq t \leq s \leq 1 .\end{cases}
\end{aligned}
$$

Proof From Lemma 2.3 we have

$$
u(t)=-I_{0+}^{\alpha} \nu(t)+c_{1} t^{\alpha-1}+c_{2} t^{\alpha-2}+c_{3} t^{\alpha-3}, \quad \text { for } c_{i} \in \mathbb{R}, i=1,2,3 .
$$

Note that $u(0)=u^{\prime}(0)=0$, and we obtain $c_{2}=c_{3}=0$. Then we have

$$
u(t)=-I_{0+}^{\alpha} v(t)+c_{1} t^{\alpha-1}=-\int_{0}^{t} \frac{(t-s)^{\alpha-1}}{\Gamma(\alpha)} v(s) d s+c_{1} t^{\alpha-1}
$$

Therefore, from the condition $u^{\prime}(1)=\int_{0}^{1} g(t) u^{\prime}(t) d t$, we have the equation

$$
-\int_{0}^{1} \frac{(1-s)^{\alpha-2}}{\Gamma(\alpha)} v(s) d s+c_{1}=-\int_{0}^{1} g(t) \int_{0}^{t} \frac{(t-s)^{\alpha-2}}{\Gamma(\alpha)} v(s) d s d t+c_{1} \int_{0}^{1} g(t) t^{\alpha-2} d t .
$$

Solving this, we have

$$
\begin{aligned}
c_{1} & =\frac{1}{1-\int_{0}^{1} g(t) t^{\alpha-2} d t}\left[\int_{0}^{1} \frac{(1-s)^{\alpha-2}}{\Gamma(\alpha)} v(s) d s-\int_{0}^{1} g(t) \int_{0}^{t} \frac{(t-s)^{\alpha-2}}{\Gamma(\alpha)} v(s) d s d t\right] \\
& =\frac{1}{1-\int_{0}^{1} g(t) t^{\alpha-2} d t}\left[\int_{0}^{1} \frac{(1-s)^{\alpha-2}}{\Gamma(\alpha)} v(s) d s-\int_{0}^{1} g(t) \int_{s}^{1} \frac{(t-s)^{\alpha-2}}{\Gamma(\alpha)} v(s) d t d s\right] .
\end{aligned}
$$

As a result, we obtain

$$
\begin{aligned}
u(t)= & -\int_{0}^{t} \frac{(t-s)^{\alpha-1}}{\Gamma(\alpha)} v(s) d s+\frac{1}{1-\int_{0}^{1} g(t) t^{\alpha-2} d t} \int_{0}^{1} \frac{t^{\alpha-1}(1-s)^{\alpha-2}}{\Gamma(\alpha)} v(s) d s \\
& -\frac{t^{\alpha-1}}{1-\int_{0}^{1} g(t) t^{\alpha-2} d t} \int_{0}^{1} g(t) \int_{s}^{1} \frac{(t-s)^{\alpha-2}}{\Gamma(\alpha)} v(s) d t d s \\
= & -\int_{0}^{t} \frac{(t-s)^{\alpha-1}}{\Gamma(\alpha)} v(s) d s+\int_{0}^{1} \frac{t^{\alpha-1}(1-s)^{\alpha-2}}{\Gamma(\alpha)} v(s) d s-\int_{0}^{1} \frac{t^{\alpha-1}(1-s)^{\alpha-2}}{\Gamma(\alpha)} v(s) d s \\
& +\frac{1}{1-\int_{0}^{1} g(t) t^{\alpha-2} d t} \int_{0}^{1} \frac{t^{\alpha-1}(1-s)^{\alpha-2}}{\Gamma(\alpha)} v(s) d s \\
& -\frac{t^{\alpha-1}}{1-\int_{0}^{1} g(t) t^{\alpha-2} d t} \int_{0}^{1} g(t) \int_{s}^{1} \frac{(t-s)^{\alpha-2}}{\Gamma(\alpha)} v(s) d t d s \\
= & \int_{0}^{1} \widetilde{G}_{1}(t, s) v(s) d s+\frac{t^{\alpha-1}}{1-\int_{0}^{1} g(t) t^{\alpha-2} d t} \int_{0}^{1} \int_{0}^{1} g(t) \widetilde{G}_{2}(t, s) d t v(s) d s \\
= & \int_{0}^{1} G_{1}(t, s) v(s) d s .
\end{aligned}
$$

This completes the proof. 
For convenience, let

$$
\begin{aligned}
G_{2}(t, s) & =\frac{\partial}{\partial t} G_{1}(t, s) \\
& =(\alpha-1)\left[\widetilde{G}_{2}(t, s)+\frac{t^{\alpha-2}}{1-\int_{0}^{1} g(t) t^{\alpha-2} d t}+\int_{0}^{1} g(t) \widetilde{G}_{2}(t, s) d t\right], \quad \text { for } t, s \in[0,1]
\end{aligned}
$$

Lemma 2.5 Suppose that (H0) holds. If $\alpha, \beta, f$ are as in (1.1), then the fractional boundary value problem (1.1) is equivalent to the Hammerstein integral equation

$$
v(t)=\int_{0}^{1} H_{1}(t, s) f\left(s, \int_{0}^{1} G_{1}(s, \tau) v(\tau) d \tau, \int_{0}^{1} G_{2}(s, \tau) v(\tau) d \tau, v(s)\right) d s,
$$

where

$$
\begin{aligned}
& H_{1}(t, s)=\widetilde{H}_{1}(t, s)+\frac{t^{\beta-1}}{1-\int_{0}^{1} h(t) t^{\beta-2} d t} \int_{0}^{1} h(t) \widetilde{H}_{2}(t, s) d t, \\
& \tilde{H}_{1}(t, s)=\frac{1}{\Gamma(\beta)} \begin{cases}t^{\beta-1}(1-s)^{\beta-2}-(t-s)^{\beta-1}, & 0 \leq s \leq t \leq 1, \\
t^{\beta-1}(1-s)^{\beta-2}, & 0 \leq t \leq s \leq 1,\end{cases} \\
& \tilde{H}_{2}(t, s)=\frac{1}{\Gamma(\beta)} \begin{cases}t^{\beta-2}(1-s)^{\beta-2}-(t-s)^{\beta-2}, & 0 \leq s \leq t \leq 1, \\
t^{\beta-2}(1-s)^{\beta-2}, & 0 \leq t \leq s \leq 1 .\end{cases}
\end{aligned}
$$

Proof Substituting $-D_{0+}^{\alpha} u=v$ into (1.1), we have

$$
\left\{\begin{array}{l}
-D_{0+}^{\beta} v(t)=f\left(t, \int_{0}^{1} G_{1}(t, s) v(s) d s, \int_{0}^{1} G_{2}(t, s) v(s) d s, v(t)\right), \quad 0<t<1 \\
v(0)=v^{\prime}(0)=0, \quad v^{\prime}(1)=\int_{0}^{1} h(t) v^{\prime}(t) d t
\end{array}\right.
$$

Using $\widetilde{f}(t)$ to replace $f(t, \cdot, \cdot, \cdot)$, and from Lemma 2.3 we obtain

$$
v(t)=-I_{0_{+}}^{\beta} \widetilde{f}(t)+c_{1} t^{\beta-1}+c_{2} t^{\beta-2}+c_{3} t^{\beta-3}, \quad \text { for } c_{i} \in \mathbb{R}, i=1,2,3 .
$$

Note that $v(0)=v^{\prime}(0)=0$ implies $c_{2}=c_{3}=0$. Hence,

$$
v(t)=-\int_{0}^{t} \frac{(t-s)^{\beta-1}}{\Gamma(\beta)} \tilde{f}(s) d s+c_{1} t^{\beta-1} .
$$

From the condition $v^{\prime}(1)=\int_{0}^{1} h(t) v^{\prime}(t) d t$, we get

$$
-\int_{0}^{1} \frac{(1-s)^{\beta-2}}{\Gamma(\beta)} \tilde{f}(s) d s+c_{1}=-\int_{0}^{1} h(t) \int_{0}^{t} \frac{(t-s)^{\beta-2}}{\Gamma(\beta)} \tilde{f}(s) d s d t+c_{1} \int_{0}^{1} h(t) t^{\beta-2} d t .
$$

Solving this equation, we obtain

$$
\begin{aligned}
c_{1}= & \frac{1}{1-\int_{0}^{1} h(t) t^{\beta-2} d t} \int_{0}^{1} \frac{(1-s)^{\beta-2}}{\Gamma(\beta)} \widetilde{f}(s) d s \\
& -\frac{1}{1-\int_{0}^{1} h(t) t^{\beta-2} d t} \int_{0}^{1} h(t) \int_{0}^{t} \frac{(t-s)^{\beta-2}}{\Gamma(\beta)} \widetilde{f}(s) d s d t
\end{aligned}
$$




$$
\begin{aligned}
= & \frac{1}{1-\int_{0}^{1} h(t) t^{\beta-2} d t} \int_{0}^{1} \frac{(1-s)^{\beta-2}}{\Gamma(\beta)} \widetilde{f}(s) d s \\
& -\frac{1}{1-\int_{0}^{1} h(t) t^{\beta-2} d t} \int_{0}^{1} h(t) \int_{s}^{1} \frac{(t-s)^{\beta-2}}{\Gamma(\beta)} \widetilde{f}(s) d t d s .
\end{aligned}
$$

Consequently, we have

$$
\begin{aligned}
v(t)= & -\int_{0}^{t} \frac{(t-s)^{\beta-1}}{\Gamma(\beta)} \widetilde{f}(s) d s+\frac{1}{1-\int_{0}^{1} h(t) t^{\beta-2} d t} \int_{0}^{1} \frac{t^{\beta-1}(1-s)^{\beta-2}}{\Gamma(\beta)} \widetilde{f}(s) d s \\
& -\frac{t^{\beta-1}}{1-\int_{0}^{1} h(t) t^{\beta-2} d t} \int_{0}^{1} h(t) \int_{s}^{1} \frac{(t-s)^{\beta-2}}{\Gamma(\beta)} \widetilde{f}(s) d t d s \\
= & -\int_{0}^{t} \frac{(t-s)^{\beta-1}}{\Gamma(\beta)} \widetilde{f}(s) d s+\int_{0}^{1} \frac{t^{\beta-1}(1-s)^{\beta-2}}{\Gamma(\beta)} \widetilde{f}(s) d s-\int_{0}^{1} \frac{t^{\beta-1}(1-s)^{\beta-2}}{\Gamma(\beta)} \widetilde{f}(s) d s \\
& +\frac{1}{1-\int_{0}^{1} h(t) t^{\beta-2} d t} \int_{0}^{1} \frac{t^{\beta-1}(1-s)^{\beta-2}}{\Gamma(\beta)} \widetilde{f}(s) d s \\
& -\frac{t^{\beta-1}}{1-\int_{0}^{1} h(t) t^{\beta-2} d t} \int_{0}^{1} h(t) \int_{s}^{1} \frac{(t-s)^{\beta-2}}{\Gamma(\beta)} \widetilde{f}(s) d t d s \\
= & \int_{0}^{1} \widetilde{H}_{1}(t, s) \widetilde{f}(s) d s+\frac{t^{\beta-1}}{1-\int_{0}^{1} h(t) t^{\beta-2} d t} \int_{0}^{1} \int_{0}^{1} h(t) \widetilde{H}_{2}(t, s) d t \tilde{f}(s) d s \\
= & \int_{0}^{1} H_{1}(t, s) \widetilde{f}(s) d s .
\end{aligned}
$$

This completes the proof.

Lemma 2.6 The functions $G_{i}, H_{1}(i=1,2)$ satisfy the properties:

(i) $G_{i}(t, s), H_{1}(t, s) \geq 0$ for $t, s \in[0,1] \times[0,1]$,

(ii) $t^{\beta-1} \phi_{\beta}(s) \leq H_{1}(t, s) \leq \phi_{\beta}(s)$ for $t, s \in[0,1] \times[0,1]$, where

$$
\phi_{\beta}(s)=\frac{s(1-s)^{\beta-2}}{\Gamma(\beta)}+\frac{\int_{0}^{1} h(t) \widetilde{H}_{2}(t, s) d t}{1-\int_{0}^{1} h(t) t^{\beta-2} d t}, s \in[0,1] .
$$

Proof We only prove (ii). From [20] we have

$$
t^{\beta-1} \frac{1}{\Gamma(\beta)} s(1-s)^{\beta-2} \leq \widetilde{H}_{1}(t, s) \leq \frac{1}{\Gamma(\beta)} s(1-s)^{\beta-2}, \quad \text { for } t, s \in[0,1] \times[0,1] .
$$

Combining this with (2.5), we easily obtain the inequalities in (ii). This completes the proof.

Let $E:=C[0,1],\|v\|:=\max _{t \in[0,1]}|v(t)|$ (here $\left.v \in E\right), P:=\{v \in E: v(t) \geq 0, \forall t \in[0,1]\}$. Then $(E,\|\cdot\|)$ is a real Banach space, and $P$ is a cone on $E$. Now, we define an operator $A: E \rightarrow E$ as follows:

$$
(A v)(t):=\int_{0}^{1} H_{1}(t, s) f\left(s, \int_{0}^{1} G_{1}(s, \tau) v(\tau) d \tau, \int_{0}^{1} G_{2}(s, \tau) v(\tau) d \tau, v(s)\right) d s
$$

for all $v \in E$. Moreover, we note that the continuity of $G_{1}, G_{2}, H_{1}$ and $f$ implies that $A$ : $E \rightarrow E$ is a completely continuous operator. Note that the existence of solutions of (2.6) is 
equivalent to that of fixed points of $A$, and then from (1.1) and (2.6) $\left(-D_{0+}^{\alpha} u=v\right)$, we see that if there exists a $\bar{y} \in E$ such that $A \bar{y}=\bar{y}$, then $\bar{y}$ is a solution for (1.1). Therefore, in what follows we study the existence of fixed points of $A$. For this purpose we need to define a linear operator $L_{a, b, c}: E \rightarrow E$ as follows:

$$
\left(L_{a, b, c} v\right)(t):=\int_{0}^{1} H_{a, b, c}(t, s) v(s) d s, \quad \forall v \in E,
$$

where $H_{a, b, c}(t, s):=a H_{3}(t, s)+b H_{2}(t, s)+c H_{1}(t, s), \forall t, s \in[0,1]$, with $a, b, c \geq 0$ and $a^{2}+b^{2}+$ $c^{2} \neq 0$; here

$$
H_{2}(t, s)=\int_{0}^{1} H_{1}(t, \tau) G_{2}(\tau, s) d \tau, \quad H_{3}(t, s)=\int_{0}^{1} H_{1}(t, \tau) G_{1}(\tau, s) d \tau, \quad \forall t, s \in[0,1] .
$$

Moreover, we know that the continuity of $H_{i}(i=1,2,3)$ implies that $L_{a, b, c}$ is a completely continuous operator and $L_{a, b, c}(P) \subset P$. Let $r\left(L_{a, b, c}\right)$ denote the spectral radius of $L_{a, b, c}$, and from Gelfand's theorem we see that $r\left(L_{a, b, c}\right)>0$ (the proof is standard; see [20, Lemma 5]).

Let $P_{0}=\left\{v \in P: v(t) \geq t^{\beta-1}\|v\|, \forall t \in[0,1]\right\}$. Then if we define an operator $\left(A_{1} v\right)(t)=$ $\int_{0}^{1} H_{1}(t, s) v(s) d s$, where $H_{1}$ is defined by (2.5), and from Lemma 2.6(ii) we have

$$
A_{1}(P) \subset P_{0} .
$$

Indeed, if $v \in P$, Lemma 2.6(ii) implies that

$$
\int_{0}^{1} t^{\beta-1} \phi_{\beta}(s) v(s) d s \leq \int_{0}^{1} H_{1}(t, s) v(s) d s \leq \int_{0}^{1} \phi_{\beta}(s) v(s) d s,
$$

and

$$
\left(A_{1} v\right)(t) \geq t^{\beta-1}\left\|A_{1} v\right\|
$$

Lemma 2.7 (see [53, Theorem 19.3]) Let $P$ be a reproducing cone in a real Banach space $E$ and let $L: E \rightarrow E$ be a compact linear operator with $L(P) \subset P$. Let $r(L)$ be the spectral radius of $L$. If $r(L)>0$, then there exists $\varphi \in P \backslash\{0\}$ such that $L \varphi=r(L) \varphi$.

Therefore, from Lemma 2.7 we see that there exists $\varphi_{a, b, c} \in P \backslash\{0\}$ such that

$$
L_{a, b, c} \varphi_{a, b, c}=r\left(L_{a, b, c}\right) \varphi_{a, b, c} .
$$

In what follows, we prove that

$$
\varphi_{a, b, c} \in P_{0} .
$$

Indeed, from (2.10) we have

$$
\begin{aligned}
\varphi_{a, b, c}(t) & =\frac{1}{r\left(L_{a, b, c}\right)}\left(L_{a, b, c} \varphi_{a, b, c}\right)(t)=\frac{1}{r\left(L_{a, b, c}\right)} \int_{0}^{1} H_{a, b, c}(t, s) \varphi_{a, b, c}(s) d s \\
& =\frac{1}{r\left(L_{a, b, c}\right)} \int_{0}^{1}\left[a H_{3}(t, s)+b H_{2}(t, s)+c H_{1}(t, s)\right] \varphi_{a, b, c}(s) d s, \quad t \in[0,1] .
\end{aligned}
$$


Using Lemma 2.6(ii) and the definitions of $H_{i}(i=1,2,3)$, we have

$$
\begin{aligned}
\left\|\varphi_{a, b, c}\right\| \leq & \frac{1}{r\left(L_{a, b, c}\right)} \int_{0}^{1}\left[a \int_{0}^{1} \phi_{\beta}(\tau) G_{1}(\tau, s) d \tau+b \int_{0}^{1} \phi_{\beta}(\tau) G_{2}(\tau, s) d \tau+c \phi_{\beta}(s)\right] \\
& \times \varphi_{a, b, c}(s) d s
\end{aligned}
$$

and

$$
\begin{aligned}
\varphi_{a, b, c}(t) \geq & \frac{1}{r\left(L_{a, b, c}\right)} \int_{0}^{1}\left[a \int_{0}^{1} t^{\beta-1} \phi_{\beta}(\tau) G_{1}(\tau, s) d \tau+b \int_{0}^{1} t^{\beta-1} \phi_{\beta}(\tau) G_{2}(\tau, s) d \tau\right. \\
& \left.+c t^{\beta-1} \phi_{\beta}(s)\right] \varphi_{a, b, c}(s) d s \\
\geq & t^{\beta-1}\left\|\varphi_{a, b, c}\right\| .
\end{aligned}
$$

Therefore, $(2.11)$ is true.

Lemma 2.8 (see [54]) Let E be a Banach space and $\Omega$ a bounded open set in E. Suppose that $A: \Omega \rightarrow E$ is a continuous compact operator. If there exists $u_{0} \in E \backslash\{0\}$ such that

$$
u-A u \neq \mu u_{0}, \quad \forall u \in \partial \Omega, \mu \geq 0,
$$

then the topological degree $\operatorname{deg}(I-A, \Omega, 0)=0$.

Lemma 2.9 (see [54]) Let E be a Banach space and $\Omega$ a bounded open set in $E$ with $0 \in \Omega$. Suppose that $A: \Omega \rightarrow E$ is a continuous compact operator. If

$$
A u \neq \mu u, \quad \forall u \in \partial \Omega, \mu \geq 1,
$$

then the topological degree $\operatorname{deg}(I-A, \Omega, 0)=1$.

\section{Main results}

Let $\alpha_{i}, \beta_{i}, \gamma_{i} \geq 0(i=1,2)$ with $\alpha_{1}^{2}+\beta_{1}^{2}+\gamma_{1}^{2} \neq 0, \alpha_{2}^{2}+\beta_{2}^{2}+\gamma_{2}^{2} \neq 0$, and $r^{-1}\left(L_{\alpha_{i}, \beta_{i}, \gamma_{i}}\right)=\lambda_{\alpha_{i}, \beta_{i}, \gamma_{i}}$ for $i=1,2$. Now, we list our assumptions for $f$ as follows:

(H1) $f \in C\left([0,1] \times \mathbb{R}^{3}, \mathbb{R}\right)$.

(H2) There exist two nonnegative functions $b(t), c(t) \in C[0,1]$ with $c(t) \not \equiv 0$ and a function $K\left(x_{1}, x_{2}, x_{3}\right) \in C\left[\mathbb{R}^{3}, \mathbb{R}^{+}\right]$such that

$$
f\left(t, x_{1}, x_{2}, x_{3}\right) \geq-b(t)-c(t) K\left(x_{1}, x_{2}, x_{3}\right), \quad \forall x_{i} \in \mathbb{R}, t \in[0,1], i=1,2,3 .
$$

(H3) $\lim _{\alpha_{1}\left|x_{1}\right|+\beta_{1}\left|x_{2}\right|+\gamma_{1}\left|x_{3}\right| \rightarrow+\infty} \frac{K\left(x_{1}, x_{2}, x_{3}\right)}{\alpha_{1}\left|x_{1}\right|+\beta_{1}\left|x_{2}\right|+\gamma_{1}\left|x_{3}\right|}=0$.

(H4) $\liminf _{\alpha_{1}\left|x_{1}\right|+\beta_{1}\left|x_{2}\right|+\gamma_{1}\left|x_{3}\right| \rightarrow+\infty} \frac{f\left(t, x_{1}, x_{2}, x_{3}\right)}{\alpha_{1}\left|x_{1}\right|+\beta_{1}\left|x_{2}\right|+\gamma_{1}\left|x_{3}\right|}>\lambda_{\alpha_{1}, \beta_{1}, \gamma_{1}}$, uniformly for $t \in[0,1]$.

(H5) $\limsup _{\alpha_{2}\left|x_{1}\right|+\beta_{2}\left|x_{2}\right|+\gamma_{2}\left|x_{3}\right| \rightarrow 0} \frac{\left|f\left(t, x_{1}, x_{2}, x_{3}\right)\right|}{\alpha_{2}\left|x_{1}\right|+\beta_{2}\left|x_{2}\right|+\gamma_{2}\left|x_{3}\right|}<\lambda_{\alpha_{2}, \beta_{2}, \gamma_{2}}$, uniformly for $t \in[0,1]$. We now present our main result.

Theorem 3.1 Suppose that (H0)-(H5) hold. Then (1.1) has at least one nontrivial solution. 
Proof From (H4) there exist $\varepsilon_{0}>0$ and $X_{0}>0$ such that

$$
\begin{gathered}
f\left(t, x_{1}, x_{2}, x_{3}\right) \geq\left(\lambda_{\alpha_{1}, \beta_{1}, \gamma_{1}}+\varepsilon_{0}\right)\left(\alpha_{1}\left|x_{1}\right|+\beta_{1}\left|x_{2}\right|+\gamma_{1}\left|x_{3}\right|\right), \\
\forall t \in[0,1], \alpha_{1}\left|x_{1}\right|+\beta_{1}\left|x_{2}\right|+\gamma_{1}\left|x_{3}\right|>X_{0} .
\end{gathered}
$$

For any given $\varepsilon$ with $\varepsilon_{0}-\|c\| \varepsilon>0$, and from (H3) there exists $X_{1}>X_{0}$ such that

$$
K\left(x_{1}, x_{2}, x_{3}\right) \leq \varepsilon\left(\alpha_{1}\left|x_{1}\right|+\beta_{1}\left|x_{2}\right|+\gamma_{1}\left|x_{3}\right|\right), \quad \forall \alpha_{1}\left|x_{1}\right|+\beta_{1}\left|x_{2}\right|+\gamma_{1}\left|x_{3}\right|>X_{1} .
$$

It follows from (H2), (3.1), (3.2) that

$$
\begin{aligned}
f\left(t, x_{1}, x_{2}, x_{3}\right) \geq & \left(\lambda_{\alpha_{1}, \beta_{1}, \gamma_{1}}+\varepsilon_{0}\right)\left(\alpha_{1}\left|x_{1}\right|+\beta_{1}\left|x_{2}\right|+\gamma_{1}\left|x_{3}\right|\right)-b(t)-c(t) K\left(x_{1}, x_{2}, x_{3}\right) \\
\geq & \left(\lambda_{\alpha_{1}, \beta_{1}, \gamma_{1}}+\varepsilon_{0}\right)\left(\alpha_{1}\left|x_{1}\right|+\beta_{1}\left|x_{2}\right|+\gamma_{1}\left|x_{3}\right|\right) \\
& -b(t)-\varepsilon c(t)\left(\alpha_{1}\left|x_{1}\right|+\beta_{1}\left|x_{2}\right|+\gamma_{1}\left|x_{3}\right|\right) \\
\geq & \left(\lambda_{\alpha_{1}, \beta_{1}, \gamma_{1}}+\varepsilon_{0}-\varepsilon c(t)\right)\left(\alpha_{1}\left|x_{1}\right|+\beta_{1}\left|x_{2}\right|+\gamma_{1}\left|x_{3}\right|\right)-b(t) \\
\geq & \left(\lambda_{\alpha_{1}, \beta_{1}, \gamma_{1}}+\varepsilon_{0}-\varepsilon\|c\|\right)\left(\alpha_{1}\left|x_{1}\right|+\beta_{1}\left|x_{2}\right|+\gamma_{1}\left|x_{3}\right|\right) \\
& -\|b\|, \quad \forall \alpha_{1}\left|x_{1}\right|+\beta_{1}\left|x_{2}\right|+\gamma_{1}\left|x_{3}\right|>X_{1} .
\end{aligned}
$$

Let $C_{X_{1}}=\left(\lambda_{\alpha_{1}, \beta_{1}, \gamma_{1}}+\varepsilon_{0}-\varepsilon\|c\|\right) X_{1}+\max _{0 \leq t \leq 1, \alpha_{1}\left|x_{1}\right|+\beta_{1}\left|x_{2}\right|+\gamma_{1}\left|x_{3}\right| \leq X_{1}}\left|f\left(t, x_{1}, x_{2}, x_{3}\right)\right|, K^{*}=$ $\max _{\alpha_{1}\left|x_{1}\right|+\beta_{1}\left|x_{2}\right|+\gamma_{1}\left|x_{3}\right| \leq X_{1}} K\left(x_{1}, x_{2}, x_{3}\right)$. Then it easy to see that

$$
\begin{aligned}
f\left(t, x_{1}, x_{2}, x_{3}\right) \geq & \left(\lambda_{\alpha_{1}, \beta_{1}, \gamma_{1}}+\varepsilon_{0}-\varepsilon\|c\|\right)\left(\alpha_{1}\left|x_{1}\right|+\beta_{1}\left|x_{2}\right|+\gamma_{1}\left|x_{3}\right|\right) \\
& -b(t)-C_{X_{1}}, \quad \forall\left(t, x_{1}, x_{2}, x_{3}\right) \in[0,1] \times \mathbb{R}^{3} .
\end{aligned}
$$

Note that $\varepsilon$ can be chosen arbitrarily small, and we let

$$
\begin{aligned}
R> & \max \left\{\frac{\left(\|b\|+\|c\| K^{*}+C_{X_{1}}\right) \int_{0}^{1} \phi_{h}(s) d s}{1-\varepsilon M_{\alpha_{1}, \beta_{1}, \gamma_{1}}\|c\|},\right. \\
& \left.\frac{\left(\lambda_{\alpha_{1}, \beta_{1}, \gamma_{1}}+2 \varepsilon_{0}-2\|c\| \varepsilon\right)\left(\|b\|+\|c\| K^{*}+C_{X_{1}}\right) \int_{0}^{1} \phi_{h}(s) d s}{\varepsilon_{0}-\|c\| \varepsilon-\varepsilon\|c\| M_{\alpha_{1}, \beta_{1}, \gamma_{1}}\left(\lambda_{\alpha_{1}, \beta_{1}, \gamma_{1}}+2 \varepsilon_{0}-2\|c\| \varepsilon\right)}\right\},
\end{aligned}
$$

where $M_{\alpha_{1}, \beta_{1}, \gamma_{1}}=\int_{0}^{1} \phi_{h}(s)\left(\alpha_{1} \int_{0}^{1} G_{1}(s, \tau) d \tau+\beta_{1} \int_{0}^{1} G_{2}(s, \tau) d \tau+\gamma_{1}\right) d s$, and $\phi_{h}(s)=\frac{(1-s)^{\beta-2}}{\Gamma(\beta)}+$ $\frac{\int_{0}^{1} h(t) \widetilde{H}_{2}(t, s) d t}{1-\int_{0}^{1} h(t) t^{\beta-2} d t}, s \in[0,1]$.

Now we prove that

$$
v-A v \neq \mu \varphi_{\alpha_{1}, \beta_{1}, \gamma_{1}}, \quad \forall v \in \partial B_{R}, \mu \geq 0,
$$

where $\varphi_{\alpha_{1}, \beta_{1}, \gamma_{1}}$ is the positive eigenfunction of $L_{\alpha_{1}, \beta_{1}, \gamma_{1}}$ corresponding to the eigenvalue $\lambda_{\alpha_{1}, \beta_{1}, \gamma_{1}}$, and then $\varphi_{\alpha_{1}, \beta_{1}, \gamma_{1}}=\lambda_{\alpha_{1}, \beta_{1}, \gamma_{1}} L_{\alpha_{1}, \beta_{1}, \gamma_{1}} \varphi_{\alpha_{1}, \beta_{1}, \gamma_{1}}$ and $\varphi_{\alpha_{1}, \beta_{1}, \gamma_{1}} \in P_{0}$ by (2.11).

Suppose (3.5) is not true. Then there exists $v_{0} \in \partial B_{R}$ and $\mu_{0}>0$ such that

$$
\nu_{0}-A v_{0}=\mu_{0} \varphi_{\alpha_{1}, \beta_{1}, \gamma_{1}} .
$$


Let

$$
\begin{aligned}
\widetilde{v}(t)= & \int_{0}^{1} H_{1}(t, s)\left[b(s)+c(s) K\left(\int_{0}^{1} G_{1}(s, \tau) v_{0}(\tau) d \tau, \int_{0}^{1} G_{2}(s, \tau) v_{0}(\tau) d \tau, v_{0}(s)\right)\right. \\
& \left.+C_{X_{1}}\right] d s, \quad \forall t \in[0,1] .
\end{aligned}
$$

Then we have

$$
\begin{aligned}
\widetilde{v}(t) \leq & \int_{0}^{1} H_{1}(t, s)\left[b(s)+c(s)\left[\varepsilon \left(\alpha_{1}\left|\int_{0}^{1} G_{1}(s, \tau) v_{0}(\tau) d \tau\right|\right.\right.\right. \\
& \left.\left.\left.+\beta_{1}\left|\int_{0}^{1} G_{2}(s, \tau) v_{0}(\tau) d \tau\right|+\gamma_{1}\left|v_{0}(s)\right|\right)+K^{*}\right]+C_{X_{1}}\right] d s \\
\leq & \int_{0}^{1} t^{\beta-1} \phi_{h}(s)\left[b(s)+c(s)\left[\varepsilon \left(\alpha_{1} \int_{0}^{1} G_{1}(s, \tau)\left|v_{0}(\tau)\right| d \tau\right.\right.\right. \\
& \left.\left.\left.+\beta_{1} \int_{0}^{1} G_{2}(s, \tau)\left|v_{0}(\tau)\right| d \tau+\gamma_{1}\left|v_{0}(s)\right|\right)+K^{*}\right]+C_{X_{1}}\right] d s \\
\leq & t^{\beta-1} \int_{0}^{1} \phi_{h}(s)\left[\|b\|+\|c\|\left[\varepsilon \left(\alpha_{1} \int_{0}^{1} G_{1}(s, \tau) d \tau\right.\right.\right. \\
& \left.\left.\left.+\beta_{1} \int_{0}^{1} G_{2}(s, \tau) d \tau+\gamma_{1}\right)\left\|v_{0}\right\|+K^{*}\right]+C_{X_{1}}\right] d s \\
\leq & t^{\beta-1}\left(\|b\|+\|c\| K^{*}+C_{X_{1}}\right) \int_{0}^{1} \phi_{h}(s) d s \\
& +t^{\beta-1} \varepsilon\|c\| \int_{0}^{1} \phi_{h}(s)\left(\alpha_{1} \int_{0}^{1} G_{1}(s, \tau) d \tau+\beta_{1} \int_{0}^{1} G_{2}(s, \tau) d \tau+\gamma_{1}\right)\left\|v_{0}\right\| d s \\
\leq & \left(\|b\|+\|c\| K^{*}+C_{X_{1}}\right) \int_{0}^{1} \phi_{h}(s) d s \\
& +\varepsilon\|c\| R \int_{0}^{1} \phi_{h}(s)\left(\alpha_{1} \int_{0}^{1} G_{1}(s, \tau) d \tau+\beta_{1} \int_{0}^{1} G_{2}(s, \tau) d \tau+\gamma_{1}\right) d s .
\end{aligned}
$$

Consequently, we have

$$
\begin{aligned}
\|\widetilde{v}\| \leq & \left(\|b\|+\|c\| K^{*}+C_{X_{1}}\right) \int_{0}^{1} \phi_{h}(s) d s \\
& +\varepsilon\|c\| R \int_{0}^{1} \phi_{h}(s)\left(\alpha_{1} \int_{0}^{1} G_{1}(s, \tau) d \tau+\beta_{1} \int_{0}^{1} G_{2}(s, \tau) d \tau+\gamma_{1}\right) d s \\
& <R .
\end{aligned}
$$

Note that $\widetilde{v} \in P_{0}$, and then from (2.9), $\varphi_{\alpha_{1}, \beta_{1}, \gamma_{1}} \in P_{0}$, and

$$
\begin{aligned}
v_{0}(t)+\widetilde{v}(t) & =A v_{0}(t)+\mu_{0} \varphi_{\alpha_{1}, \beta_{1}, \gamma_{1}}(t)+\widetilde{v}(t) \\
& =\int_{0}^{1} H_{1}(t, s)\left[f\left(s, \int_{0}^{1} G_{1}(s, \tau) v_{0}(\tau) d \tau, \int_{0}^{1} G_{2}(s, \tau) v_{0}(\tau) d \tau, v_{0}(s)\right)\right.
\end{aligned}
$$


Fu et al. Journal of Inequalities and Applications

(2019) 2019:104

Page 11 of 16

$$
\begin{aligned}
& \left.+b(s)+c(s) K\left(\int_{0}^{1} G_{1}(s, \tau) v_{0}(\tau) d \tau, \int_{0}^{1} G_{2}(s, \tau) v_{0}(\tau) d \tau, v_{0}(s)\right)+C_{X_{1}}\right] d s \\
& +\mu_{0} \varphi_{\alpha_{1}, \beta_{1}, \gamma_{1}}(t)
\end{aligned}
$$

we have

$$
v_{0}+\widetilde{v} \in P_{0}
$$

using the fact that

$$
\begin{aligned}
& f\left(s, \int_{0}^{1} G_{1}(s, \tau) v_{0}(\tau) d \tau, \int_{0}^{1} G_{2}(s, \tau) v_{0}(\tau) d \tau, v_{0}(s)\right)+b(s) \\
& \quad+c(s) K\left(\int_{0}^{1} G_{1}(s, \tau) v_{0}(\tau) d \tau, \int_{0}^{1} G_{2}(s, \tau) v_{0}(\tau) d \tau, v_{0}(s)\right)+C_{X_{1}} \in P .
\end{aligned}
$$

Therefore, (2.10), (3.4) and (3.7) enable us to obtain

$$
\begin{aligned}
& A v_{0}(t)+\widetilde{v}(t) \\
& =\int_{0}^{1} H_{1}(t, s) f\left(s, \int_{0}^{1} G_{1}(s, \tau) v_{0}(\tau) d \tau, \int_{0}^{1} G_{2}(s, \tau) v_{0}(\tau) d \tau, v_{0}(s)\right) d s \\
& +\int_{0}^{1} H_{1}(t, s)\left[b(s)+c(s) K\left(\int_{0}^{1} G_{1}(s, \tau) v_{0}(\tau) d \tau, \int_{0}^{1} G_{2}(s, \tau) v_{0}(\tau) d \tau, v_{0}(s)\right)\right. \\
& \left.+C_{X_{1}}\right] d s \\
& \geq\left(\lambda_{\alpha_{1}, \beta_{1}, \gamma_{1}}+\varepsilon_{0}-\varepsilon\|c\|\right) \int_{0}^{1} H_{1}(t, s)\left[\alpha_{1}\left|\int_{0}^{1} G_{1}(s, \tau) v_{0}(\tau) d \tau\right|\right. \\
& \left.+\beta_{1}\left|\int_{0}^{1} G_{2}(s, \tau) v_{0}(\tau) d \tau\right|+\gamma_{1}\left|v_{0}(s)\right|\right] d s \\
& -\int_{0}^{1} H_{1}(t, s)\left[b(s)+C_{X_{1}}\right] d s \\
& +\int_{0}^{1} H_{1}(t, s)\left[b(s)+c(s) K\left(\int_{0}^{1} G_{1}(s, \tau) v_{0}(\tau) d \tau, \int_{0}^{1} G_{2}(s, \tau) v_{0}(\tau) d \tau, v_{0}(s)\right)\right. \\
& \left.+C_{X_{1}}\right] d s \\
& \geq\left(\lambda_{\alpha_{1}, \beta_{1}, \gamma_{1}}+\varepsilon_{0}-\varepsilon\|c\|\right) \int_{0}^{1} H_{1}(t, s)\left[\alpha_{1}\left|\int_{0}^{1} G_{1}(s, \tau) \nu_{0}(\tau) d \tau\right|\right. \\
& \left.+\beta_{1}\left|\int_{0}^{1} G_{2}(s, \tau) v_{0}(\tau) d \tau\right|+\gamma_{1}\left|v_{0}(s)\right|\right] d s \\
& \geq\left(\lambda_{\alpha_{1}, \beta_{1}, \gamma_{1}}+\varepsilon_{0}-\varepsilon\|c\|\right) \mid \alpha_{1} \int_{0}^{1} H_{1}(t, s) \int_{0}^{1} G_{1}(s, \tau) \nu_{0}(\tau) d \tau d s \\
& +\beta_{1} \int_{0}^{1} H_{1}(t, s) \int_{0}^{1} G_{2}(s, \tau) v_{0}(\tau) d \tau d s+\gamma_{1} \int_{0}^{1} H_{1}(t, s) v_{0}(s) d s \\
& \geq\left(\lambda_{\alpha_{1}, \beta_{1}, \gamma_{1}}+\varepsilon_{0}-\varepsilon\|c\|\right) \mid \alpha_{1} \int_{0}^{1} H_{3}(t, \tau) \nu_{0}(\tau) d \tau+\beta_{1} \int_{0}^{1} H_{2}(t, \tau) \nu_{0}(\tau) d \tau
\end{aligned}
$$




$$
\begin{aligned}
& +\gamma_{1} \int_{0}^{1} H_{1}(t, s) v_{0}(s) d s \mid \\
= & \left(\lambda_{\alpha_{1}, \beta_{1}, \gamma_{1}}+\varepsilon_{0}-\varepsilon\|c\|\right)\left|\int_{0}^{1} H_{\alpha_{1}, \beta_{1}, \gamma_{1}}(t, s) v_{0}(s) d s\right| \\
\geq & \left(\lambda_{\alpha_{1}, \beta_{1}, \gamma_{1}}+\varepsilon_{0}-\varepsilon\|c\|\right) \int_{0}^{1} H_{\alpha_{1}, \beta_{1}, \gamma_{1}}(t, s) v_{0}(s) d s .
\end{aligned}
$$

From the definition of operator $L_{\alpha_{1}, \beta_{1}, \gamma_{1}}$, we get

$$
\begin{aligned}
& \left(\lambda_{\alpha_{1}, \beta_{1}, \gamma_{1}}+\varepsilon_{0}-\|c\| \varepsilon\right) \int_{0}^{1} H_{\alpha_{1}, \beta_{1}, \gamma_{1}}(t, s) v_{0}(s) d s \\
& =\lambda_{\alpha_{1}, \beta_{1}, \gamma_{1}} \int_{0}^{1} H_{\alpha_{1}, \beta_{1}, \gamma_{1}}(t, s)\left(v_{0}(s)+\widetilde{v}(s)\right) d s+\left(\varepsilon_{0}-\|c\| \varepsilon\right) \int_{0}^{1} H_{\alpha_{1}, \beta_{1}, \gamma_{1}}(t, s) v_{0}(s) d s \\
& \quad-\lambda_{\alpha_{1}, \beta_{1}, \gamma_{1}} \int_{0}^{1} H_{\alpha_{1}, \beta_{1}, \gamma_{1}}(t, s) \widetilde{v}(s) d s \\
& =\lambda_{\alpha_{1}, \beta_{1}, \gamma_{1}} L_{\alpha_{1}, \beta_{1}, \gamma_{1}}\left(v_{0}+\widetilde{v}\right)(t)+\left(\varepsilon_{0}-\|c\| \varepsilon\right) \int_{0}^{1} H_{\alpha_{1}, \beta_{1}, \gamma_{1}}(t, s)\left(v_{0}(s)+\widetilde{v}(s)\right) d s \\
& \quad-\left(\lambda_{\alpha_{1}, \beta_{1}, \gamma_{1}}+\varepsilon_{0}-\|c\| \varepsilon\right) \int_{0}^{1} H_{\alpha_{1}, \beta_{1}, \gamma_{1}}(t, s) \widetilde{v}(s) d s .
\end{aligned}
$$

From (3.10), we get $v_{0}(t)+\widetilde{v}(t) \geq t^{\beta-1}\left\|v_{0}+\widetilde{v}\right\| \geq t^{\beta-1}\left(\left\|v_{0}\right\|-\|\widetilde{v}\|\right), t \in[0,1]$, and hence from (3.8) we have

$$
\begin{aligned}
\left(\varepsilon_{0}-\right. & \|c\| \varepsilon) \int_{0}^{1} H_{\alpha_{1}, \beta_{1}, \gamma_{1}}(t, s)\left(v_{0}(s)+\widetilde{v}(s)\right) d s \\
& -\left(\lambda_{\alpha_{1}, \beta_{1}, \gamma_{1}}+\varepsilon_{0}-\|c\| \varepsilon\right) \int_{0}^{1} H_{\alpha_{1}, \beta_{1}, \gamma_{1}}(t, s) \widetilde{v}(s) d s \\
\geq & \left(\varepsilon_{0}-\|c\| \varepsilon\right)(R-\|\widetilde{v}\|) \int_{0}^{1} s^{\beta-1} H_{\alpha_{1}, \beta_{1}, \gamma_{1}}(t, s) d s-\left(\lambda_{\alpha_{1}, \beta_{1}, \gamma_{1}}+\varepsilon_{0}-\|c\| \varepsilon\right) \\
& \times\left[\left(\|b\|+\|c\| K^{*}+C_{X_{1}}\right) \int_{0}^{1} \phi_{h}(s) d s+\varepsilon\|c\| R M_{\alpha_{1}, \beta_{1}, \gamma_{1}}\right] \int_{0}^{1} s^{\beta-1} H_{\alpha_{1}, \beta_{1}, \gamma_{1}}(t, s) d s \\
\geq & 0
\end{aligned}
$$

Combining (3.11), (3.12) and (3.13), and we obtain

$$
A v_{0}(t)+\widetilde{v}(t) \geq \lambda_{\alpha_{1}, \beta_{1}, \gamma_{1}} L_{\alpha_{1}, \beta_{1}, \gamma_{1}}\left(v_{0}+\widetilde{v}\right)(t), \quad t \in[0,1]
$$

Therefore, using (3.6) and (3.14) we have

$$
v_{0}+\widetilde{v}=A v_{0}+\mu_{0} \varphi_{\alpha_{1}, \beta_{1}, \gamma_{1}}+\widetilde{v} \geq \lambda_{\alpha_{1}, \beta_{1}, \gamma_{1}} L_{\alpha_{1}, \beta_{1}, \gamma_{1}}\left(v_{0}+\widetilde{v}\right)+\mu_{0} \varphi_{\alpha_{1}, \beta_{1}, \gamma_{1}} \geq \mu_{0} \varphi_{\alpha_{1}, \beta_{1}, \gamma_{1}}
$$

Define

$$
\mu^{*}=\sup \left\{\mu>0: v_{0}+\widetilde{v} \geq \mu \varphi_{\alpha_{1}, \beta_{1}, \gamma_{1}}\right\}
$$


It is easy to see that $\mu^{*} \geq \mu_{0}$ and $v_{0}+\widetilde{v} \geq \mu^{*} \varphi_{\alpha_{1}, \beta_{1}, \gamma_{1}}$. From $\varphi_{\alpha_{1}, \beta_{1}, \gamma_{1}}=\lambda_{\alpha_{1}, \beta_{1}, \gamma_{1}} L_{\alpha_{1}, \beta_{1}, \gamma_{1}} \times$ $\varphi_{\alpha_{1}, \beta_{1}, \gamma_{1}}$, we obtain

$$
\lambda_{\alpha_{1}, \beta_{1}, \gamma_{1}} L_{\alpha_{1}, \beta_{1}, \gamma_{1}}\left(v_{0}+\widetilde{v}\right) \geq \lambda_{\alpha_{1}, \beta_{1}, \gamma_{1}} L_{\alpha_{1}, \beta_{1}, \gamma_{1}} \mu^{*} \varphi_{\alpha_{1}, \beta_{1}, \gamma_{1}}=\mu^{*} \varphi_{\alpha_{1}, \beta_{1}, \gamma_{1}}
$$

Hence

$$
v_{0}+\widetilde{v} \geq \lambda_{\alpha_{1}, \beta_{1}, \gamma_{1}} L_{\alpha_{1}, \beta_{1}, \gamma_{1}}\left(v_{0}+\widetilde{v}\right)+\mu_{0} \varphi_{\alpha_{1}, \beta_{1}, \gamma_{1}} \geq\left(\mu_{0}+\mu^{*}\right) \varphi_{\alpha_{1}, \beta_{1}, \gamma_{1}}
$$

which contradicts the definition of $\mu^{*}$. Therefore, (3.5) holds, and from Lemma 2.8 we obtain

$$
\operatorname{deg}\left(I-A, B_{R}, 0\right)=0 .
$$

From (H5) there exist $0<\varepsilon_{1}<\lambda_{\alpha_{2}, \beta_{2}, \gamma_{2}}$ and $0<r<R$ such that

$$
\left|f\left(t, x_{1}, x_{2}, x_{3}\right)\right| \leq\left(\lambda_{\alpha_{2}, \beta_{2}, \gamma_{2}}-\varepsilon_{1}\right)\left(\alpha_{2}\left|x_{1}\right|+\beta_{2}\left|x_{2}\right|+\gamma_{2}\left|x_{3}\right|\right),
$$

for all $x_{i} \in \mathbb{R}, i=1,2,3, t \in[0,1]$ with $0 \leq \alpha_{2}\left|x_{1}\right|+\beta_{2}\left|x_{2}\right|+\gamma_{2}\left|x_{3}\right|<r$. Consequently, we obtain

$$
\begin{aligned}
|(A v)(t)| \leq & \left(\lambda_{\alpha_{2}, \beta_{2}, \gamma_{2}}-\varepsilon_{1}\right) \int_{0}^{1} H_{1}(t, s)\left(\alpha_{2}\left|\int_{0}^{1} G_{1}(s, \tau) v(\tau) d \tau\right|\right. \\
& \left.+\beta_{2}\left|\int_{0}^{1} G_{2}(s, \tau) v(\tau) d \tau\right|+\gamma_{2}|v(s)|\right) d s \\
\leq & \left(\lambda_{\alpha_{2}, \beta_{2}, \gamma_{2}}-\varepsilon_{1}\right) \int_{0}^{1} H_{1}(t, s)\left(\alpha_{2} \int_{0}^{1} G_{1}(s, \tau)|v(\tau)| d \tau\right. \\
& \left.+\beta_{2} \int_{0}^{1} G_{2}(s, \tau)|v(\tau)| d \tau+\gamma_{2}|v(s)|\right) d s \\
= & \left(\lambda_{\alpha_{2}, \beta_{2}, \gamma_{2}}-\varepsilon_{1}\right) \int_{0}^{1} H_{\alpha_{2}, \beta_{2}, \gamma_{2}}(t, s)|v(s)| d s \\
= & \left(\lambda_{\alpha_{2}, \beta_{2}, \gamma_{2}}-\varepsilon_{1}\right)\left(L_{\alpha_{2}, \beta_{2}, \gamma_{2}}|v|\right)(t), \quad \forall t \in[0,1], v \in E,\|v\| \leq r .
\end{aligned}
$$

Now for this $r$, we prove that

$$
A v \neq \lambda v, \quad \forall v \in \partial B_{r}, \lambda \geq 1
$$

Assume the contrary. Then there exist $v_{0} \in \partial B_{r}$ and $\lambda_{0} \geq 1$ such that $A v_{0}=\lambda_{0} v_{0}$. Let $\omega(t)=$ $\left|v_{0}(t)\right|$. Then $\omega \in \partial B_{r} \cap P$ and

$$
\omega \leq \frac{1}{\lambda_{0}}\left(\lambda_{\alpha_{2}, \beta_{2}, \gamma_{2}}-\varepsilon_{1}\right) L_{\alpha_{2}, \beta_{2}, \gamma_{2}} \omega \leq\left(\lambda_{\alpha_{2}, \beta_{2}, \gamma_{2}}-\varepsilon_{1}\right) L_{\alpha_{2}, \beta_{2}, \gamma_{2}} \omega .
$$

By induction, we have $\omega \leq\left(\lambda_{\alpha_{2}, \beta_{2}, \gamma_{2}}-\varepsilon_{1}\right)^{n} L_{\alpha_{2}, \beta_{2}, \gamma_{2}}^{n} \omega$, for $n=1,2, \ldots$. As a result, we have

$$
\|\omega\| \leq\left(\lambda_{\alpha_{2}, \beta_{2}, \gamma_{2}}-\varepsilon_{1}\right)^{n}\left\|L_{\alpha_{2}, \beta_{2}, \gamma_{2}}^{n}\right\|\|\omega\|
$$


and thus

$$
1 \leq\left(\lambda_{\alpha_{2}, \beta_{2}, \gamma_{2}}-\varepsilon_{1}\right)^{n}\left\|L_{\alpha_{2}, \beta_{2}, \gamma_{2}}^{n}\right\| .
$$

Therefore, by Gelfand's theorem, we have

$$
\left(\lambda_{\alpha_{2}, \beta_{2}, \gamma_{2}}-\varepsilon_{1}\right) r\left(L_{\alpha_{2}, \beta_{2}, \gamma_{2}}\right)=\left(\lambda_{\alpha_{2}, \beta_{2}, \gamma_{2}}-\varepsilon_{1}\right) \lim _{n \rightarrow \infty} \sqrt[n]{\left\|L_{\alpha_{2}, \beta_{2}, \gamma_{2}}^{n}\right\|} \geq 1 .
$$

This contradicts

$$
\left(\lambda_{\alpha_{2}, \beta_{2}, \gamma_{2}}-\varepsilon_{1}\right) r\left(L_{\alpha_{2}, \beta_{2}, \gamma_{2}}\right)=1-\varepsilon_{1} r\left(L_{\alpha_{2}, \beta_{2}, \gamma_{2}}\right)<1 .
$$

Thus (3.16) holds and from Lemma 2.9 we have

$$
\operatorname{deg}\left(I-A, B_{r}, 0\right)=1 .
$$

Now (3.15) and (3.17) imply that

$$
\operatorname{deg}\left(I-A, B_{R} \backslash \bar{B}_{r}, 0\right)=\operatorname{deg}\left(I-A, B_{R}, 0\right)-\operatorname{deg}\left(I-A, B_{r}, 0\right)=-1 .
$$

Therefore the operator $A$ has at least one fixed point in $B_{R} \backslash \bar{B}_{r}$. Equivalently, (1.1) has at least one nontrivial solution. This completes the proof.

\section{Acknowledgements}

Not applicable.

\section{Funding}

Research supported by the National Natural Science Foundation of China (Grant No. 11601048), Natural Science Foundation of Chongqing (Grant No. cstc2016jcyjA0181), the Science and Technology Research Program of Chongqing Municipal Education Commission (Grant No. KJQN201800533), Natural Science Foundation of Chongqing Normal University (Grant No. 16XYY24)

\section{Availability of data and materials}

Not applicable.

\section{Competing interests}

The authors declare that they have no competing interests.

\section{Authors' contributions}

The authors conceived of the study, drafted the manuscript, and approved the final manuscript.

\section{Author details}

${ }^{1}$ College of Mathematics and System Sciences, Shandong University of Science and Technology, Qingdao, China. ${ }^{2}$ School of Mathematical Sciences, Chongqing Normal University, Chongqing, China. ${ }^{3}$ School of Mathematics, Statistics and Applied Mathematics, National University of Ireland, Galway, Ireland.

\section{Publisher's Note}

Springer Nature remains neutral with regard to jurisdictional claims in published maps and institutional affiliations.

Received: 8 February 2019 Accepted: 8 April 2019 Published online: 16 April 2019

\section{References}

1. Kilbas, A., Srivastava, H., Trujillo, J.: Theory and Applications of Fractional Differential Equations. North-Holland Mathematics Studies, vol. 204. Elsevier, Amsterdam (2006)

2. Podlubny, I.: Fractional Differential Equations. Mathematics in Science and Engineering, vol. 198. Academic Press, San Diego (1999)

3. Samko, S., Kilbas, A., Marichev, O.: Fractional Integrals and Derivatives: Theory and Applications. Gordon \& Breach, Yverdon (1993) 
4. Jiang, J., Liu, L., Wu, Y.: Positive solutions to singular fractional differential system with coupled boundary conditions, Commun. Nonlinear Sci. Numer. Simul. 18(11), 3061-3074 (2013)

5. Wang, Y., Liu, L., Zhang, X., Wu, Y.: Positive solutions of an abstract fractional semipositone differential system model for bioprocesses of HIV infection. Appl. Math. Comput. 258, 312-324 (2015)

6. Bai, Z:: On positive solutions of a nonlocal fractional boundary value problem. Nonlinear Anal. 72, 916-924 (2010)

7. Wang, Y., Liu, L., Wu, Y.: Positive solutions for a nonlocal fractional differential equation. Nonlinear Anal. 74(11), 3599-3605 (2011)

8. Cui, Y:: Uniqueness of solution for boundary value problems for fractional differential equations. Appl. Math. Lett. 51 48-54 (2016)

9. Wang, Y., Liu, L.: Positive solutions for a class of fractional infinite-point boundary value problems. Bound. Value Probl. 2018, Article ID 118 (2018)

10. Liu, X., Liu, L., Wu, Y.: Existence of positive solutions for a singular nonlinear fractional differential equation with integral boundary conditions involving fractional derivatives. Bound. Value Probl. 2018, Article ID 24 (2018)

11. Jiang, J., Liu, W., Wang, H.: Positive solutions for higher order nonlocal fractional differential equation with integral boundary conditions. J. Funct. Spaces 2018, Article ID 6598351 (2018)

12. Hao, X., Wang, H., Liu, L., Cui, Y.: Positive solutions for a system of nonlinear fractional nonlocal boundary value problems with parameters and p-Laplacian operator. Bound. Value Probl. 2017, Article ID 182 (2017)

13. Zuo, M., Hao, X., Liu, L., Cui, Y.: Existence results for impulsive fractional integro-differential equation of mixed type with constant coefficient and antiperiodic boundary conditions. Bound. Value Probl. 2017, Article ID 161 (2017)

14. Zhang, X., Liu, L., Zou, Y.: Fixed-point theorems for systems of operator equations and their applications to the fractional differential equations. J. Funct. Spaces 2018, Article ID 7469868 (2018)

15. Wu, J., Zhang, X., Liu, L., Wu, Y., Cui, Y.: The convergence analysis and error estimation for unique solution of a p-Laplacian fractional differential equation with singular decreasing nonlinearity. Bound. Value Probl. 2018, Article ID $82(2018)$

16. Zhang, X., Wu, J., Liu, L., Wu, Y., Cui, Y.: Convergence analysis of iterative scheme and error estimation of positive solution for a fractional differential equation. Math. Model. Anal. 23(4), 611-626 (2018)

17. He, J., Zhang, X., Liu, L., Wu, Y., Cui, Y.: Existence and asymptotic analysis of positive solutions for a singular fractional differential equation with nonlocal boundary conditions. Bound. Value Probl. 2018, Article ID 189 (2018)

18. Zhang, X., Liu, L., Wu, Y., Zou, Y.: Existence and uniqueness of solutions for systems of fractional differential equations with Riemann-Stieltjes integral boundary condition. Adv. Differ. Equ. 2018, Article ID 204 (2018)

19. Liu, B., Li, J., Liu, L., Wang, Y.: Existence and uniqueness of nontrivial solutions to a system of fractional differential equations with Riemann-Stieltjes integral conditions. Adv. Differ. Equ. 2018. Article ID 306 (2018)

20. Xu, J., Wei, Z:: Positive solutions for a class of fractional boundary value problems. Nonlinear Anal., Model. Control 21(1), 1-17 (2016)

21. Pu, R., Zhang, X., Cui, Y., Li, P., Wang, W.: Positive solutions for singular semipositone fractional differential equation subject to multipoint boundary conditions. J. Funct. Spaces 2017, Article ID 5892616 (2017)

22. Guo, Y: Nontrivial solutions for boundary-value problems of nonlinear fractional differential equations. Bull. Korean Math. Soc. 47(1), 81-87 (2010)

23. Zhang, K.: On a sign-changing solution for some fractional differential equations. Bound. Value Probl. 2017, Article ID 59 (2017)

24. Cui, Y., Ma, W., Sun, Q., Su, X.: New uniqueness results for boundary value problem of fractional differential equation. Nonlinear Anal., Model. Control 23(1), 31-39 (2018)

25. Zou, Y., He, G.: On the uniqueness of solutions for a class of fractional differential equations. Appl. Math. Lett. 74, 68-73 (2017)

26. Zhang, K., Wang, J., Ma, W.: Solutions for integral boundary value problems of nonlinear Hadamard fractional differential equations. J. Funct. Spaces 2018, Article ID 2193234 (2018)

27. Zhang, Y.: Existence results for a coupled system of nonlinear fractional multi-point boundary value problems at resonance. J. Inequal. Appl. 2018, Article ID 198 (2018)

28. Ma, W., Meng, S., Cui, Y.: Resonant integral boundary value problems for Caputo fractional differential equations. Math. Probl. Eng. 2018, Article ID 5438592 (2018)

29. Ma, W., Cui, Y.: The eigenvalue problem for Caputo type fractional differential equation with Riemann-Stieltjes integral boundary conditions. J. Funct. Spaces 2018, Article ID 2176809 (2018)

30. Sun, Q., Meng, S., Cui, Y.: Existence results for fractional order differential equation with nonlocal Erdélyi-Kober and generalized Riemann-Liouville type integral boundary conditions at resonance. Adv. Differ. Equ. 2018, Article ID 243 (2018)

31. Zhong, Q., Zhang, X., Lu, X., Fu, Z.: Uniqueness of successive positive solution for nonlocal singular higher-order fractional differential equations involving arbitrary derivatives. J. Funct. Spaces 2018, Article ID 6207682 (2018)

32. Dong, X., Bai, Z., Zhang, S.: Positive solutions to boundary value problems of $p$-Laplacian with fractional derivative. Bound. Value Probl. 2017, Article ID 5 (2017)

33. Song, Q., Bai, Z:: Positive solutions of fractional differential equations involving the Riemann-Stieltjes integral boundary condition. Adv. Differ. Equ. 2018, Article ID 183 (2018)

34. Zhang, X., Liu, L., Wiwatanapataphee, B., Wu, Y.: The eigenvalue for a class of singular $p$-Laplacian fractional differential equations involving the Riemann-Stieltjes integral boundary condition. Appl. Math. Comput. 235, 412-422 (2014)

35. Qi, T., Liu, Y., Cui, Y.: Existence of solutions for a class of coupled fractional differential systems with nonlocal boundary conditions. J. Funct. Spaces 2017. Article ID 6703860 (2017)

36. Zhang, L., Sun, Z., Hao, X.: Positive solutions for a singular fractional nonlocal boundary value problem. Adv. Differ. Equ. 2018, Article ID 381 (2018)

37. Yan, F., Zuo, M., Hao, X.: Positive solution for a fractional singular boundary value problem with $p$-Laplacian operator. Bound. Value Probl. 2018, Article ID 51 (2018)

38. Fan, W., Hao, X., Liu, L., Wu, Y.: Nontrivial solutions of singular fourth-order Sturm-Liouville boundary value problems with a sign-changing nonlinear term. Appl. Math. Comput. 217(15), 6700-6708 (2011)

39. Hao, X., Zuo, M., Liu, L.: Multiple positive solutions for a system of impulsive integral boundary value problems with sign-changing nonlinearities. Appl. Math. Lett. 82, 24-31 (2018) 
40. Liu, L., Liu, B., Wu, Y.: Nontrivial solutions for higher-order $m$-point boundary value problem with a sign-changing nonlinear term. Appl. Math. Comput. 217(8), 3792-3800 (2010)

41. Zhang, K., O'Regan, D., Fu, Z.: Nontrivial solutions for boundary value problems of a fourth order difference equation with sign-changing nonlinearity. Adv. Differ. Equ. 2018, Article ID 370 (2018)

42. Li, H., Sun, J.: Positive solutions of superlinear semipositone nonlinear boundary value problems. Comput. Math. Appl. 61(9), 2806-2815 (2011)

43. Zhao, Z.: Positive solutions of semi-positone Hammerstein integral equations and applications. Appl. Math. Comput. 219(5), 2789-2797 (2012)

44. Liu, J., Zhao, Z.: Multiple positive solutions for second-order three-point boundary-value problems with sign changing nonlinearities. Electron. J. Differ. Equ. 2012, Article ID 152 (2012)

45. Zhao, Z: Existence of positive solutions for $2 n$ th-order singular semipositone differential equations with Sturm-Liouville boundary conditions. Nonlinear Anal. 72(3-4), 1348-1357 (2010)

46. Lin, X., Zhao, Z.: Sign-changing solution for a third-order boundary-value problem in ordered Banach space with lattice structure. Bound. Value Probl. 2014, Article ID 132 (2014)

47. Wu, Y., Zhao, Z.: Positive solutions for third-order boundary value problems with change of signs. Appl. Math. Comput. 218(6), 2744-2749 (2011)

48. Zhang, K.: Nontrivial solutions of fourth-order singular boundary value problems with sign-changing nonlinear terms. Topol. Methods Nonlinear Anal. 40(1), 53-70 (2012)

49. Cui, Y.: Computation of topological degree in ordered Banach spaces with lattice structure and applications. Appl. Math. 58(6), 689-702 (2013)

50. Cui, Y., Sun, J.: On existence of positive solutions of coupled integral boundary value problems for a nonlinear singular superlinear differential system. Electron. J. Qual. Theory Differ. Equ. 2012, Article ID 41 (2012)

51. Cui, Y., Zou, Y: An existence and uniqueness theorem for a second order nonlinear system with coupled integral boundary value conditions. Appl. Math. Comput. 256, 438-444 (2015)

52. Sun, F., Liu, L., Zhang, X., Wu, Y.: Spectral analysis for a singular differential system with integral boundary conditions. Mediterr. J. Math. 13(6), 4763-4782 (2016)

53. Deimling, K.: Nonlinear Functional Analysis. Springer, Berlin (1985)

54. Guo, D., Lakshmikantham, V.: Nonlinear Problems in Abstract Cones. Academic Press, Orlando (1988)

\section{Submit your manuscript to a SpringerOpen ${ }^{\circ}$ journal and benefit from:}

- Convenient online submission

- Rigorous peer review

- Open access: articles freely available online

- High visibility within the field

- Retaining the copyright to your article

Submit your next manuscript at $\boldsymbol{~ s p r i n g e r o p e n . c o m ~}$ 